\title{
CORRESPONDENCE
}

\section{Comments on "A Wave-Resolving Simulation of Langmuir Circulations with a Nonhydrostatic Free-Surface Model: Comparison with Craik-Leibovich Theory and an Alternative Eulerian View of the Driving Mechanism"}

\author{
GeORge MeLlor \\ Program in Atmospheric and Oceanic Sciences, Princeton University, Princeton, New Jersey
}

(Manuscript received 26 October 2018, in final form 5 February 2019)

\begin{abstract}
The results of the subject paper are reviewed wherein credible Langmuir cells are produced by a numerical solution of the primitive fluid dynamic equations with a free surface. Whereas it is a major achievement, the claim that the same results support the general application of the so-called vortex force equations is challenged.
\end{abstract}

\section{Introduction}

The subject paper (Fujiwara et al. 2018, hereafter FYM) is important. A skillful numerical design and execution and presentation of results produce recognizable Langmuir cells absent any external parameterizations. It also provides insight into the issue of the vortex force as opposed to the wave radiation stress terms in the mean momentum equation. FYM seem to favor the former for which it is convenient to repeat here their Eq. (1), which they refer to as the Craik-Leibovich equation (Craik and Leibovich 1976, hereafter CL); it features the vortex force, the second term on the right side of

$$
\frac{\partial \overline{\mathbf{u}}}{\partial t}+\overline{\mathbf{u}} \cdot \nabla \overline{\mathbf{u}}=-\nabla(\bar{p}+\Pi)+\mathbf{u}^{\mathrm{St}} \times \overline{\boldsymbol{\omega}}+\nu \nabla^{2} \overline{\mathbf{u}} .
$$

The vector $\overline{\mathbf{u}}$ is the phase-averaged Eulerian velocity, and $\bar{p}$ is here defined as the phase-averaged kinematic pressure; the other variables are defined in FYM and below. An important distinction will be made as to whether Eq. (1) is to be applied to resolved or unresolved numerical problems.

Section 2 is a brief review of FYM's analysis of their numerical results. Section 3 distinguishes between resolved and unresolved finite difference equations and

\footnotetext{
Corresponding author: Prof. George Mellor, glmellor@princeton. edu
}

solutions as defined therein. The so-called wave radiation equations are reviewed in section 4 and section 5 . Section 6 is a summary.

\section{FYM's analysis of their results}

From the primitive equation, FYM's Eq. (2), using vector identities, one can obtain Lamb's equation,

$$
\frac{\partial \mathbf{u}}{\partial t}-\mathbf{u} \times \boldsymbol{\omega}=-\nabla\left(p+\mathbf{u}^{2} / 2\right)-\mathbf{g}+\nu \nabla^{2} \mathbf{u},
$$

where the vorticity $\boldsymbol{\omega}=\nabla \times \mathbf{u} ; p$ is kinematic pressure, and is $\nu$ an eddy viscosity. Now substitute $\mathbf{u}=\overline{\mathbf{u}}+\mathbf{u}^{\prime}$, where $\overline{\mathbf{u}}$ is the phase-averaged velocity as in FYM's Eq. (5) and $\mathbf{u}^{\prime}$ is the wave orbital velocity. Similarly, $\boldsymbol{\omega}=\overline{\boldsymbol{\omega}}+\boldsymbol{\omega}^{\prime}$. Next, take the curl of the equation and phase-average so that

$$
\frac{\partial \overline{\boldsymbol{\omega}}}{\partial t}-\nabla \times \overline{\mathbf{u}} \times \overline{\boldsymbol{\omega}}-\nabla \times \overline{\overline{\mathbf{u}}^{\prime} \times \boldsymbol{\omega}^{\prime}}=\nu \nabla^{2} \overline{\boldsymbol{\omega}} .
$$

Note that Eq. (3) is relatively simple because the curl of terms related to the irrotational wave portions of Eq. (2) and the gradient of scalar terms are nil.

They then build a numerical case that

$$
\operatorname{curl}\left(\overline{\mathbf{u}^{\prime} \times \boldsymbol{\omega}^{\prime}}\right)=\operatorname{curl}\left(\mathbf{u}^{\mathrm{St}} \times \overline{\boldsymbol{\omega}}\right),
$$

where $\mathbf{u}^{\mathrm{St}}$ is Stokes drift. Equation (4) is in accordance with CL's analytical determination. Note that CL 
posited two intersecting waves, and consequently Stokes drift, progressing at small plus and minus angles relative to the primary direction, this to produce a variation in flow normal to the primary direction (i.e., cells); this has been labeled the CL1 theory. Do FYM's numerical perturbations on the primary wave of FYM's simulation produce similar intersecting waves? That is an interesting question. A second theory, the CL2 is based on instabilities derived directly from Eq. (1).

After "uncurling" Eq. (4), the result is $\left(\overline{\mathbf{u}^{\prime} \times \boldsymbol{\omega}^{\prime}}\right)=$ $\left(\mathbf{u}^{\text {St }} \times \overline{\boldsymbol{\omega}}\right)$ plus a gradient term (Mellor 2016) so that the vortex term in Eq. (1) is simplified.

\section{Resolution}

As detailed in their paper, FYM numerically solve their primitive Eqs. (2) and (3) with a free surface and forced by waves and obtain what certainly appears to be Langmuir cells. For a wave period of $10 \mathrm{~s}$, their numerical time step is $0.5 \mathrm{~s}$; for their wavelength of $156 \mathrm{~m}$, their spatial resolutions are a uniform $2.4 \mathrm{~m}$ in the $x, y$, and $z$ directions respectively.

For many numerical applications, time steps and spatial grid elements are much larger. The finite difference equations are obtained by horizontal spatial and temporal averaging necessitating introduction of parameterizations (vertical and horizontal eddy viscosities) hopefully to mimic missing small-scale motions. For the present purposes, I will term numerical solutions with small grid elements resolved solutions whereas solutions with larger grid elements are unresolved solutions, even though grid elements are often small enough to resolve important oceanic features such as western boundary currents or even eddies. Thus, the results of FYM are resolved calculations. ${ }^{1}$

Equation (1) is attributed to Leibovich (1977) and then to McWilliams and Restrepo (1999, hereafter MR). It was applied to unresolved equations and solutions (e.g., Uchiyama et al. 2010). Their strategy was to "uncurl" Eq. (3). In so doing, they insert irrotational term of their own choosing to arrive at the Eq. (1). Terms otherwise included in Eq. (2) are missing as discussed in section 5 below. For more detailed exploration of the vortex force equations versus the wave radiation stress equations, refer to Mellor (2016).

Langmuir cells are often thought to be an important contribution to vertical momentum mixing in

\footnotetext{
${ }^{1}$ They do use a viscosity characteristic of an eddy viscosity (three orders of magnitude larger than a molecular viscosity), but apparently this does not interfere with the capture of surface wave effects. Importantly, motions transverse to the main wave progression are resolved.
}

the ocean surface boundary layer. To obtain unresolved solutions in the FYM setup, define a spanwise average as $\overline{(-)}^{y}=\int_{0}^{\lambda_{10}}(\cdot) d y$. I have asked coauthor Fujiwara to obtain certain averages for which he graciously responded. A spanwise average of $\overline{u^{\prime} w^{\prime}}=0$ was obtained and is the expected result for linear waves. However, $\overline{\bar{u}}^{y}<5 \times 10^{-5} \mathrm{~m}^{2} \mathrm{~s}^{-2}$; this compares with the imposed skin friction (as distinct from form drag) wind stress of $\tau=2.0 \times 10^{-2} \mathrm{~N} \mathrm{~m}^{-2}$ or a Reynolds stress of $\tau / \rho_{w}=2.0 \times 10^{-5} \mathrm{~m}^{2} \mathrm{~s}^{-2}$. The FYM simulation is essentially swell. For reference to a wind driven flow of the same amplitude $(a=0.5 \mathrm{~m}), \tau / \rho_{w}$ is an order of magnitude larger.

\section{The wave radiation stress equations}

To return to the primitive momentum equation, let $\mathbf{u} \times \boldsymbol{\omega}=-\mathbf{u} \cdot \nabla \mathbf{u}+\nabla\left(\mathbf{u}^{2} / 2\right)$ and adding $\mathbf{u} \nabla \cdot \mathbf{u}$ to Eq. (2), one obtains the usual flux form of Eq. (2). As before, let $\mathbf{u}=\overline{\mathbf{u}}+\mathbf{u}^{\prime}$ and $p=\bar{p}+p^{\prime}$, where $\mathbf{u}^{\prime}$ and $p^{\prime}$ are the linearized wave solutions (or orbital relations) ${ }^{2}$ so that

$$
\begin{gathered}
\frac{\partial}{\partial t}\left(\overline{\mathbf{u}}+\mathbf{u}^{\prime}\right)+\nabla \cdot\left(\overline{\mathbf{u}} \overline{\mathbf{u}}+\overline{\mathbf{u}} \mathbf{u}^{\prime}+\mathbf{u}^{\prime} \overline{\mathbf{u}}+\mathbf{u}^{\prime} \mathbf{u}^{\prime}\right) \\
\quad=-\nabla\left(\bar{p}+p^{\prime}\right)-\mathbf{g}+\nu \nabla^{2}\left(\overline{\mathbf{u}}+\mathbf{u}^{\prime}\right)
\end{gathered}
$$

After phase-averaging, the term $\overline{\mathbf{u}^{\prime} \mathbf{u}^{\prime}}$ is a radiation stress term, of which more will follow. If one takes the curl of Eq. (5), the terms involving the curl of $\mathbf{u}^{\prime}$, the gradient term, and the constant $\mathbf{g}$ vanish; they are terms retained in the equations derived by Mellor $(2003,2015)$ including the wave radiation stress terms. Here we simply list the equations which invoke the boundary layer or hydrostatic approximation. Thus, the continuity equation is

$$
\frac{\partial D U_{\beta}}{\partial x_{\beta}}+\frac{\partial \Omega}{\partial \varsigma}+\frac{\partial \hat{\eta}}{\partial t}=0
$$

and momentum equation is

$$
\begin{gathered}
\frac{\partial}{\partial t}\left(D U_{\alpha}\right)+\frac{\partial}{\partial x_{\beta}}\left(D U_{\alpha} U_{\beta}+D S_{\alpha \beta}\right)+\frac{\partial \Omega U_{\alpha}}{\partial \varsigma} \\
=-\frac{\partial}{\partial x_{\alpha}}\left(g \hat{\eta}+p_{\mathrm{atm}}\right)+\frac{\partial \tau_{\alpha}}{\partial \zeta} .
\end{gathered}
$$

\footnotetext{
${ }^{2}$ In Mellor (2016), following CL and MR, a formal expansion is performed whereby $\mathbf{u}^{\prime}$ is order $\varepsilon=k a$ and $\overline{\mathbf{u}}$ is order $\varepsilon^{2} ; k a$ is the wave slope. Instead of first taking the curl of the momentum equation, I dealt with full Eq. (5). The lowest-order equations are $\nabla \cdot \mathbf{u}^{\prime}=0$ and $\partial \mathbf{u}^{\prime} / \partial t+\nabla p^{\prime}=-\mathbf{g}$, which yield the linear wave relations and are terms which vanish from Eq. (2) because of the curl operation.
} 
The above sigma coordinate equations are simplified by omitting Coriolis and baroclinic terms. The equations differentiate between the horizontal and vertical as do the linear wave equations. The sigma coordinate is $s=$ $(\bar{z}-\hat{\eta}) / D$, where $D \equiv \hat{\eta}+h$ and $\hat{\eta}$ is the mean elevation. The subscripts $\alpha$ or $\beta=(x, y)$. Note that $U_{\alpha}=\hat{u}_{\alpha}+u_{S \alpha}$, which includes the Eulerian velocity, $\overline{\mathbf{u}}=\left(\hat{u}_{\alpha}, \hat{w}\right)=$ $(\hat{u}, \hat{v}, \hat{w})$, plus Stokes drift. Parameter $\Omega$ is the nearlyvertical velocity such that $\Omega=0$ when $z=\hat{\eta}$ or $-h$. The wave radiation stress is

$S_{\alpha \beta}=\overline{\tilde{u}_{D \alpha} \tilde{u}_{D \beta}}-\delta_{\alpha \beta} \overline{\tilde{w}_{D}^{2}}+\delta_{\alpha \beta}\left[\frac{\partial}{\partial \bar{z}}\left(\overline{\tilde{p}_{D} \tilde{s}_{D}}-g \overline{\tilde{s}_{D}^{2}} / 2\right)\right]$.

The water column momentum transfer by turbulence or waves is here represented by $\tau_{\alpha}$. The system is closed by inserting the linear wave relations into Eq. (8) and empirical expressions for $\tau_{\alpha}$. When Eq. (6) and Eq. (7) are vertically integrated, the equations of Longuet-Higgins and Stewart $(1962,1964)$ are obtained.

In Eq. (8), $\tilde{u}_{\alpha}$ and $\tilde{p}$ denotes the linear wave velocity and pressure. In deriving Eqs. (7) and (8), $\mathbf{u}^{\prime}=\left(\tilde{u}_{\alpha}, \tilde{w}\right)=$ $(\tilde{u}, \tilde{v}, \tilde{w})$ span the vertical range, $\hat{\eta}+\tilde{\eta} \geq z \geq-h$, and $\tilde{\eta}$ is the instantaneous wave elevation. The free surface is explicitly recognized unlike the derivation leading to Eq. (1). The additional subscript $D$ denotes the same equations which, after phase-averaging, span $\hat{\eta} \geq \bar{z} \geq-h$. The material displacement throughout the water column is $\tilde{s}$, which can be obtained from $\tilde{w}=\partial \tilde{s} / \partial t$; at the surface $\tilde{s}=\tilde{\eta}$.

\section{The embedded vortex force}

The essential results in this section can be obtained from the sigma equations Eq. (6) and Eq. (7), but to compare with Eq. (1), convert to Cartesian coordinates (hereafter, replace $\bar{z}$ with $z$ ) as in Mellor (2005) such that the continuity equation is

$$
\frac{\partial U_{\beta}}{\partial x_{\beta}}+\frac{\partial \hat{w}}{\partial z}=0,
$$

and the momentum equation is

$$
\begin{aligned}
& \frac{\partial U_{\alpha}}{\partial t} U_{\beta} \frac{\partial U_{\alpha}}{\partial x_{\beta}}+\hat{w} \frac{\partial U_{\alpha}}{\partial z}+\frac{\partial}{\partial x_{\alpha}}\left(g \hat{\eta}+p_{\text {atm }}\right) \\
& \quad=\frac{\partial \tau_{\alpha}}{\partial z}-F_{\alpha},
\end{aligned}
$$

where the radiation stress term is complicated in Cartesian coordinates (Mellor 2005) such that,

$$
F_{\alpha} \equiv \frac{1}{D} \frac{\partial D S_{\alpha \beta}}{\partial x_{\beta}}+\frac{\partial S_{\alpha \beta}}{\partial z}\left(\frac{\partial \hat{\eta}}{\partial x_{\beta}}+\frac{z-\hat{\eta}}{D} \frac{\partial D}{\partial x_{\alpha}}\right) .
$$

Now let $U_{\alpha}=\hat{u}_{\alpha}+u_{S \alpha}$ in Eq. (10) and add to the left side

$$
\partial\left(\hat{u}_{\beta} u_{S \beta}\right) / \partial x_{\alpha}-\hat{u}_{\beta} \partial u_{S \beta} / \partial x_{\alpha}-u_{S \beta} \partial \hat{u}_{\beta} / \partial x_{\alpha}=0 .
$$

After some algebra, one obtains

$$
\begin{aligned}
& \frac{\partial \hat{u}_{\alpha}}{\partial t}+\hat{u}_{\beta} \frac{\partial \hat{u}_{\alpha}}{\partial x_{\beta}}+\hat{w} \frac{\partial \hat{u}_{\alpha}}{\partial z}+u_{S \beta}\left(\frac{\partial \hat{u}_{\alpha}}{\partial x_{\beta}}-\frac{\partial \hat{u}_{\beta}}{\partial x_{\alpha}}\right)+\frac{\partial}{\partial x_{\alpha}}\left(\hat{p}+\hat{u}_{\beta} u_{S \beta}\right)-\frac{\partial \tau_{\alpha}}{\partial z} \\
& +\hat{u}_{\beta}\left(\frac{\partial u_{S \alpha}}{\partial x_{\beta}}-\frac{\partial u_{S \beta}}{\partial x_{\alpha}}\right)+u_{S \beta} \frac{\partial u_{S \alpha}}{\partial x_{\beta}}+\frac{\partial u_{S \alpha}}{\partial t}+\hat{w} \frac{\partial u_{S_{\alpha}}}{\partial z}=-F_{\alpha} .
\end{aligned}
$$

The top line including the vortex force term conforms to the horizontal components of Eq. (1), but, as anticipated, the bottom line are terms which were excluded from Eq. (1) after the "curl-uncurl" process operated on the equations of motion. The extra terms in Eq. (12) are in accord with Smith (2006) after adding Eq. (11) and after manipulation by Mellor (2015, appendix B), although Smith dealt with vertically integrated equations.

\section{Summary}

For several decades, the complex subject of surface wave-current interaction has been a source of confusion. One reason has been the introduction of a vortex force term into the momentum equation. An important contribution to an understanding of wave-current processes is the paper by FYM. Thus, the picture that emerges is as follows:

(i) For resolved finite difference equations, FYM's numerical calculations using the primitive equations [Eqs. (2) and (3) in FYM] produce Langmuir-like cells. The FYM paper is supportive of the CL1 or CL2 analysis, such that Langmuir cells are also produced by resolved calculations of Eq. (1), although specific results are not shown. Apparently, the reduced Eq. (1) removes lowest-order terms via the curl-uncurl process, leaving higher terms that are responsible for cells. The lowest-order terms are in Eq. (12), including the wave radiation stress terms. 
(ii) For unresolved finite differencing, cells are obviously absent. Nevertheless, in MR and Uchiyama et al. (2010), for example, Eq. (1) is apparently expected to provide missing wave information. However, the dominant phase-averaged wave terms in Eq. (10) are those associated with radiation stress terms as indicated by scale analysis in FYM's section 3d and in section 8 of Mellor (2016), wherein the ratio of vortex force to radiation stress terms in Eqs. (7) or (12) are of order $\left|\hat{u}_{\alpha}\right| / c$ and where $\hat{u}$ and $c$ are the Eulerian velocity and wave phase speed, respectively. In the FYM case and in most of the real ocean, $\left|\hat{u}_{\alpha}\right| / c<10^{-2}$. Equations (6) and (7) were originally derived by Longuet-Higgins and Stewart $(1962,1964)$ for the vertically integrated equations and then derived by Mellor (2003, 2015) for the vertically dependent equations.

(iii) The perturbations due to turbulence require empirical parameterizations; however, the dominate perturbations due to waves are given analytically by $S_{\alpha \beta}$ in Eq. (7) after insertion of the linear wave terms in Eq. (7). Although not obvious, the vortex force is contained in Eq. (7), and this is demonstrated by Eq. (12). Whether or not the resolved and nonhydrostatic forms of Eqs. (6) and (7) would produce Langmuir cells have yet to be determined.

\section{REFERENCES}

Craik, A. D. D., and S. Leibovich, 1976: A rational model for Langmuir circulation. J. Fluid Mech., 73, 401-426, https:// doi.org/10.1017/S0022112076001420.
Fujiwara, Y., Y. Yoshikawa, and Y. Matsumura, 2018: A waveresolving simulation of Langmuir circulations with a nonhydrostatic free-surface model: Comparison with CraikLeibovich theory and an alternative Eulerian view of the driving mechanism. J. Phys. Oceanogr., 48, 1691-1708, https:// doi.org/10.1175/JPO-D-17-0199.1.

Leibovich, S., 1977: Convective instability of stably stratified water in the ocean. J. Fluid Mech., 82, 561-581, https://doi.org/ 10.1017/S0022112077000846.

Longuet-Higgins, M. S., and R. W. Stewart, 1962: Radiation stress and mass transport in gravity waves, with application to 'surfbeats.' J. Fluid Mech., 13, 481-504, https://doi.org/10.1017/ S0022112062000877.

— and - 1964: Radiation stresses in water waves; A physical discussion with applications. Deep-Sea Res. Oceanogr. Abstr., 11, 529-562, https://doi.org/10.1016/0011-7471(64)90001-4.

McWilliams, J. C., and J. M. Restrepo, 1999: The wave-driven ocean circulation. J. Phys. Oceanogr., 29, 2523-2540, https:// doi.org/10.1175/1520-0485(1999)029<2523:TWDOC >2.0.CO;2.

Mellor, G. L., 2003: The three dimensional, current and surface wave equations. J. Phys. Oceanogr., 33, 1978-1989, https://doi.org/ 10.1175/1520-0485(2003)033<1978:TTCASW>2.0.CO;2.

- 2005: Some consequences of the three-dimensional current and surface wave equations. J. Phys. Oceanogr., 35, 2291-2298, https://doi.org/10.1175/JPO2794.1.

- 2015: A combined derivation of the integrated and verticallyresolved, coupled wave-current equations. J. Phys. Oceanogr., 45, 1453-1463, https://doi.org/10.1175/JPO-D-14-0112.1.

- 2016: On theories dealing with the interaction of surface waves and ocean circulation. J. Geophys. Res. Oceans, 121, 4474 4486, https://doi.org/10.1002/2016JC011768.

Smith, J. A., 2006: Wave-current interactions in finite depth. J. Phys. Oceanogr., 36, 1403-1419, https://doi.org/10.1175/ JPO2911.1.

Uchiyama, Y., J. C. McWilliams, and A. F. Shchepetkin, 2010: Wavecurrent interaction in an oceanic circulation model with a vortex-force formalism: Application to the surf zone. Ocean Modell., 34, 16-35, https://doi.org/10.1016/j.ocemod.2010.04.002. 